\title{
Volume hologram replication system for photovoltaic applications
}

\section{Benjamin D. Chrysler, Raymond K. Kostuk}

Benjamin D. Chrysler, Raymond K. Kostuk, "Volume hologram replication system for photovoltaic applications," Proc. SPIE 10759, New Concepts in Solar and Thermal Radiation Conversion and Reliability, 107590W (17 September 2018); doi: 10.1117/12.2321348

SPIE Event: SPIE Optical Engineering + Applications, 2018, San Diego, California, United States 


\title{
Volume hologram replication system for photovoltaic applications
}

\author{
Benjamin D. Chrysler ${ }^{* a}$, Raymond K. Kostuk ${ }^{\mathrm{a}, \mathrm{b}}$ \\ ${ }^{A}$ University of Arizona, College of Optical Sciences, 1630 E. University Blvd., Tucson, Arizona \\ 85721, USA; ${ }^{\mathrm{B}}$ University of Arizona, Department of Electrical and Computer Engineering, 1230 \\ E. Speedway Blvd. 1230 E. Speedway Blvd., Tucson, Arizona, 85721, USA
}

\begin{abstract}
A replication system and method for fabricating volume hologram arrays is reviewed in this paper. The replication system can be used to fabricate high-efficiency transmission volume holographic lens arrays that are well-suited for spectrum-splitting photovoltaic applications. As in the well-known contact-copy replication technique, the new technique uses a master hologram to generate the desired holographic exposure, however no contact is required with the copy hologram. The object and reference beams for the holographic exposure are generated by discrete "coupling elements" on the master hologram and coupled through a prism to form an interference pattern on the copy hologram. The system can be implemented using relatively inexpensive lab equipment, but also has potential for large-scale production of holographic elements. A prototype system was developed and used to fabricate an experimental holographic lens array with a large aperture $(9.6 \mathrm{~cm} \mathrm{X} 6 \mathrm{~cm})$ and high median diffraction efficiency $(95.6 \%)$.
\end{abstract}

Keywords: holography, replication, manufacturing, spectrum splitting, Covestro Bayfol HX, array

\section{INTRODUCTION}

Spectrum-splitting photovoltaic systems (SSPV) have potential for high energy yield and overcome some of the problems faced by concentration PV systems based on series-connected multijunction cells. In theory, SSPV systems can achieve higher efficiency ${ }^{1}$ and better performance under variable illumination ${ }^{2}$ than series-connected multijunction PV systems. Many potential designs reported in literature also have practical system advantages in form factor, diffuse energy collection, and tracking system requirements.

SSPV systems attain high efficiency by using optical elements to divide the solar spectrum into spectral bands and direct each spectral band to a set of PV cells with different bandgap energies. Volume Holographic Optical Elements (VHOE) are a promising optical element for use in spectral division, due to their ability to diffract efficiently in a single order and to focus light and for their inexpensive material costs ${ }^{3}$. One implementation of VHOE's in SSPV systems uses Volume Holographic Lenses ${ }^{4,5}$ (VHL) and is notable for conversion efficiencies greater than 30\%, module thickness less than $2.5 \mathrm{~cm}$, and ability to convert diffuse sunlight. Although not included in the power conversion efficiency metric, the ability to convert diffuse sunlight is valuable because it accounts for more than 20$50 \%$ of total solar energy in most regions of the United States ${ }^{6}$. The VHL-PV system is depicted in Fig. 1 and uses a set of two PV cells with different bandgap energies. The cells are configured in rectangular strips and light is focused and dispersed onto the PV cells. Sunlight at the bandgap wavelength of the higher energy bandgap is focused to the junction of the two cells. The longer wavelength light is dispersed to the low energy bandgap while the shorter wavelengths are dispersed to the higher energy bandgap cell.

New Concepts in Solar and Thermal Radiation Conversion and Reliability, edited by Jeremy N. Munday,

Peter Bermel, Michael D. Kempe, Proc. of SPIE Vol. 10759, 107590W · C 2018 SPIE

CCC code: $0277-786 \mathrm{X} / 18 / \$ 18 \cdot$ doi: $10.1117 / 12.2321348$ 


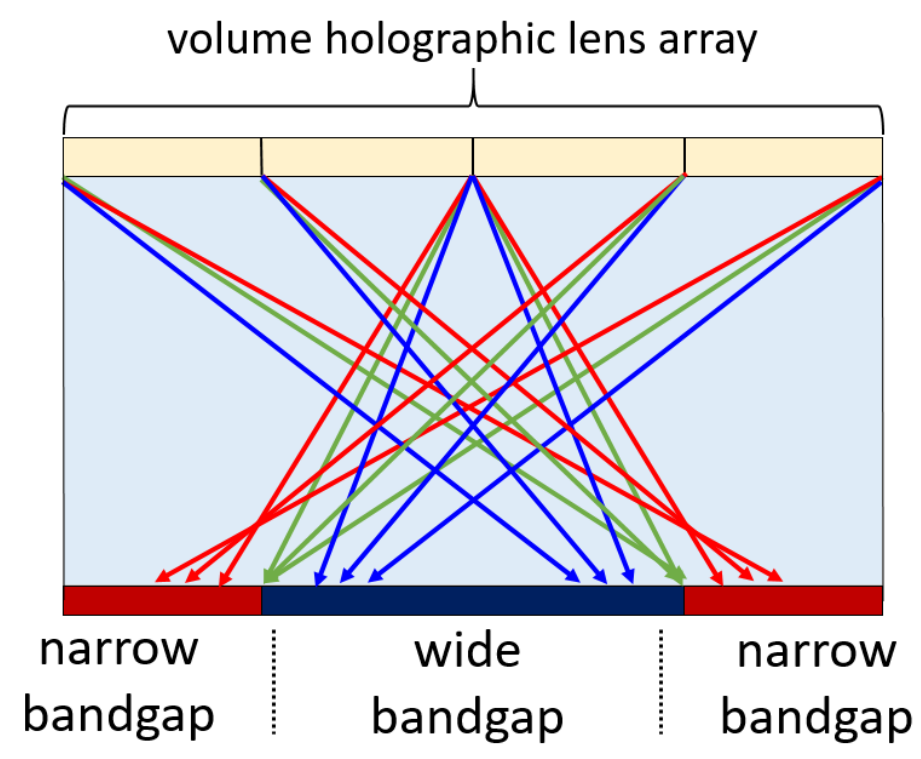

Figure 1: Illustration of volume holographic lens photovoltaic system. Light is dispersed by volume holographic lenses onto strips of PV cells with different bandgap energies.

A key challenge for SSPV systems is manufacturing of VHOEs at an industrial scale. VHOEs for PV applications must have very high diffraction efficiency $(80-90 \%)$ to achieve high PV power conversion efficiency and justify the additional cost and complexity of including the VHOE. In addition, some SSPV systems like the VHL-PV system require spatially-variant VHOEs, which are difficult to manufacture at a high volume with prior replication techniques.

A few replication systems for manufacture of VHOEs have been presented in literature ${ }^{7-11}$. Although each system has unique aspects, they are all based on variations of the well-known contact copy technique. In the contact-copy technique, a master hologram is placed in close contact with a copy hologram. Light incident on the master hologram is diffracted with $50 \%$ efficiency and interferes with the transmitted portion of the beam ${ }^{12}$. The resulting interference pattern produces a copy of the master hologram in the copy hologram. Many aspects of replication systems based on contact copy are not ideal and have problems with speed, yield, alignment, and complexity.

In this paper, we review a new "prism-coupled" replication technique ${ }^{13}$ that is unique from the contact-copy method. Although the system uses a master hologram like the contact-copy technique, direct contact is not required with the copy hologram and the system does not require a mask since the limiting aperture is recorded in the master hologram. The resulting replication system is simple and easy to align. The technique is well-suited for spatially varying object and reference beams, which makes it useful for fabricating VHL arrays. After describing the technique, a prototype replication system is presented, and a holographic lens array is reported with large dimensions $(9.6 \mathrm{~cm} \times 6 \mathrm{~cm})$, high median peak diffraction efficiency $(95.6 \%)$, and low variation in peak diffraction efficiency $(+/-0.8 \%)$.

\section{REPLICATION SYSTEM}

In this section we describe how the new prism-coupled replication technique is used to form volume hologram arrays and then we describe a technique to fabricate the master VHOE.

\subsection{Method for copying holograms}

The process used to generate the holographic exposure for forming hologram array elements is depicted in Fig. 2. The reference and object beams are generated using a "reference beam coupling element" and an "object beam coupling element" that are located in different regions of the master VHOE. The master VHOE is in optical contact 
with the prism and the diffracted beams from the coupling elements are transmitted through the long face of the prism to overlap on the surface of the copy VHOE. Light that is not diffracted is reflected on the long face of the prism by total internal reflection and filtered from the system. The copy VHOE is aligned parallel to the long face of the prism and is separated by the working distance ' $\mathrm{z}_{0}$ '. An array can be formed using a 'step and repeat' method where the copy VHOE stage is laterally translated in between a sequence of exposures as illustrated in Fig. 3.

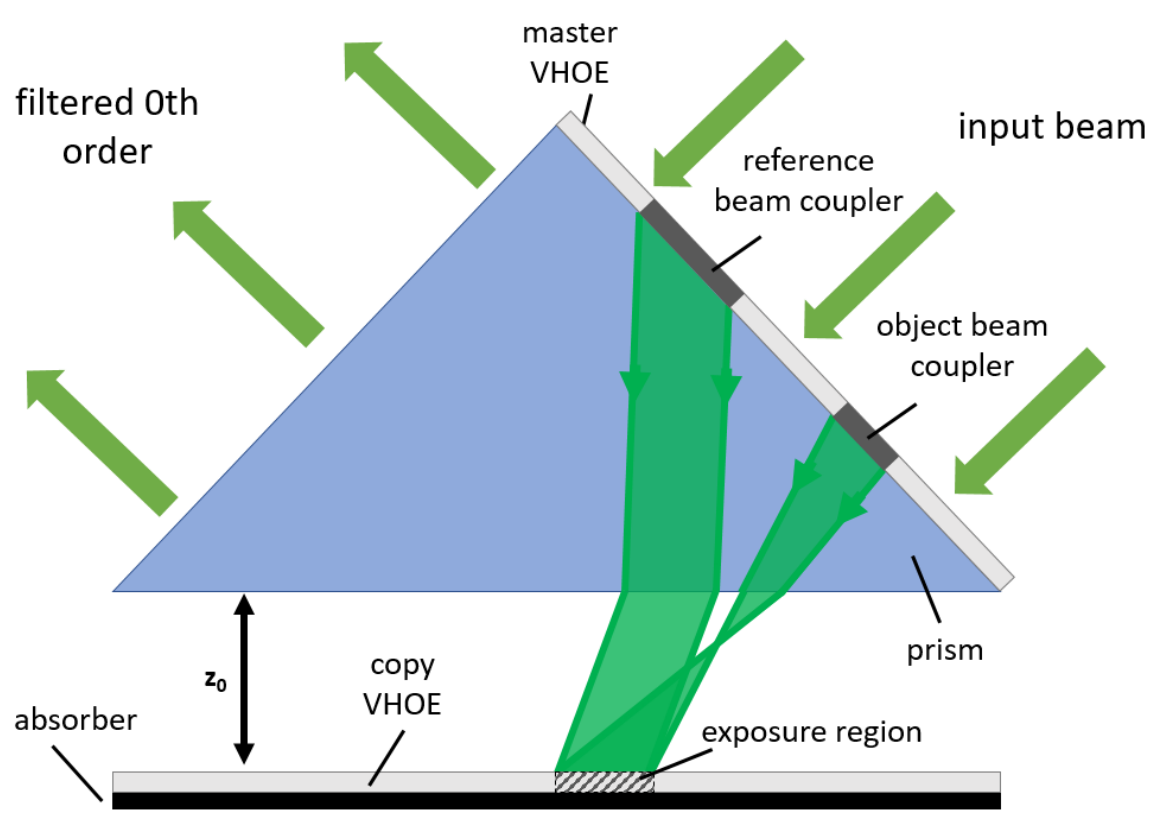

Figure 2: In the prism-coupled replication technique, object and reference beams are diffracted by coupling elements in the master VHOE to form a holographic exposure on the copy VHOE. Light that is not diffracted is filtered from the system through total internal reflection. 


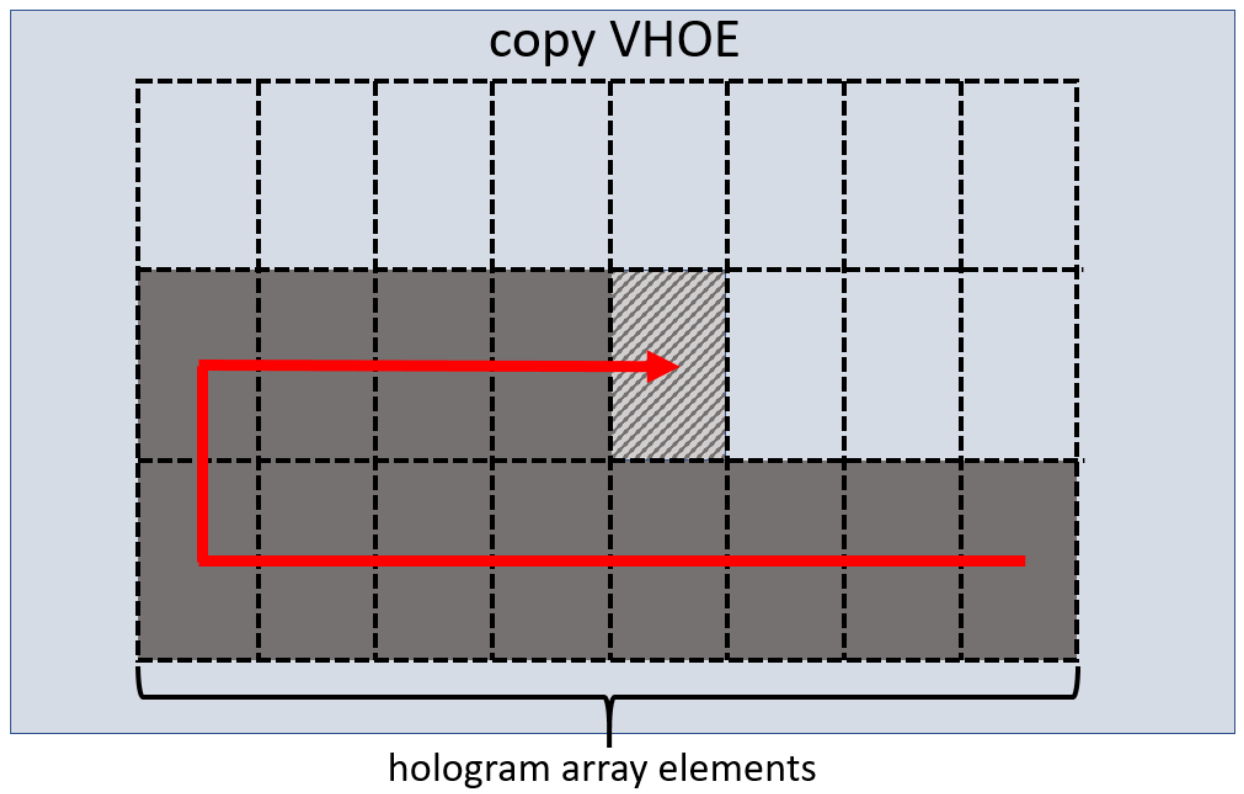

Figure 3: hologram arrays are formed by making exposures in different regions of the copy VHOE. This is accomplished using a 'step and repeat' method where the stage is laterally translated in between exposures.

\subsection{Method for fabricating master VHOE}

The master VHOE is fabricated by forming each coupling element in sequence. The recording geometry depicted in Fig. 4a is similar to the one used to fabricate the copy VHOE. An aperture with the desired dimensions of the array elements is positioned at a distance ' $\mathrm{z}_{0}$ ' from the prism. The unexposed master VHOE is placed in optical contact with one of the prism faces and an absorber. The reference beam coupling element is formed by illuminating the aperture with the conjugate reference beam which refracts through the bottom face of the prism and propagates to the master VHOE. Another prism face is illuminated with a "master reference beam" that reflects off the long face of the prism by total internal refection and impinges upon the master VHOE. This beam is limited by an adjustable window to the region where the conjugate reference beam is incident on the master VHOE. After making the exposure to form the reference beam coupling element, the object beam coupling element is formed in a similar manner as shown in Fig. 4b. The master VHOE is illuminated with the conjugate object beam and the adjustable window is altered to limit the master reference beam to the region of the object beam coupling element. After making both exposures, the master VHOE is processed and then used to copy hologram array elements. 


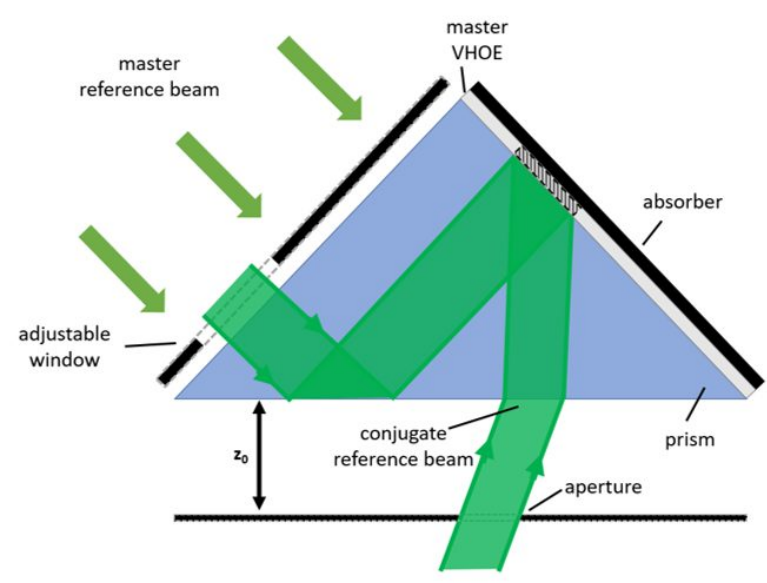

(a)

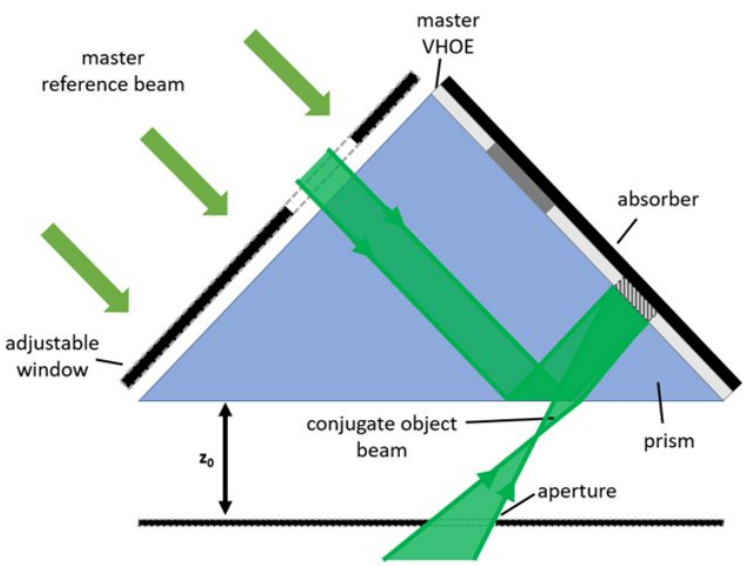

(b)

Figure 4: The optical setup for fabricating the master VHOE. The coupling elements are formed in a sequence of two holographic exposures to form the a) reference beam coupling element and (b) the object beam coupling element.

\section{DESIGN PROCEDURE}

Values for the working distance ' $\mathrm{z}_{0}$ ', aperture offset ' $\mathrm{x}_{0}$ ', and prism face length ' $\mathrm{l}$ ' are determined based on the hologram construction parameters ' $\theta_{1}$ ' and ' $\theta_{2}$ ' and cylindrical lens position ' $\mathrm{d}$ '. These parameters are depicted in Fig. 5 and are determined using the following design procedure. In this procedure, we assume the reference beam is planar and the object beam is cylindrical. This assumption is made to tailor the procedure for the VHL-PV system application, although it can easily be modified for other applications.

A. Determine desired beam angles, lens position, and aperture dimensions. The reference and object beam angles ' $\theta_{1}$ ' and ' $\theta_{2}$ ' and cylindrical lens position ' $\mathrm{d}$ ' are determined based on the desired diffraction properties for the resulting hologram and for the VHL-PV application can be determined using the technique described in references ${ }^{4,5}$. The aperture dimension ' $\mathrm{w}_{0}$ ' is set equal to the desired aperture of the resulting hologram array element.

B. Determine working distance, offset, and prism face length. Allowable values for the working distance ' $\mathrm{z}_{0}$ ', aperture offset ' $\mathrm{x}_{0}$ ', and prism face length ' $\mathrm{l}$ ' are determined based on a 'non-overlap' condition which requires that the coupling elements on the master VHOE do not overlap or lie outside the dimensions of the prism face. These values are freely adjustable within a range that satisfies the non-overlap condition and are dependent on the values determined in step ' $\mathrm{A}$ '. It can be determined whether this condition is satisfied either through experimentation or through a ray-tracing simulation.

C. Determine optimal exposure energies for the master VHOE and the copy VHOE. Exposure energies for the coupling elements ' $\mathrm{E}_{\mathrm{r}}$ ' and ' $\mathrm{E}_{\mathrm{o}}$ ' and array elements ' $\mathrm{E}_{\mathrm{c}}$ ' should be optimized independently of each other since each grating will have different spatial frequencies. They are determined by exposing trial holograms with a range of exposure energies and selecting the values that result in the highest diffraction efficiency. 


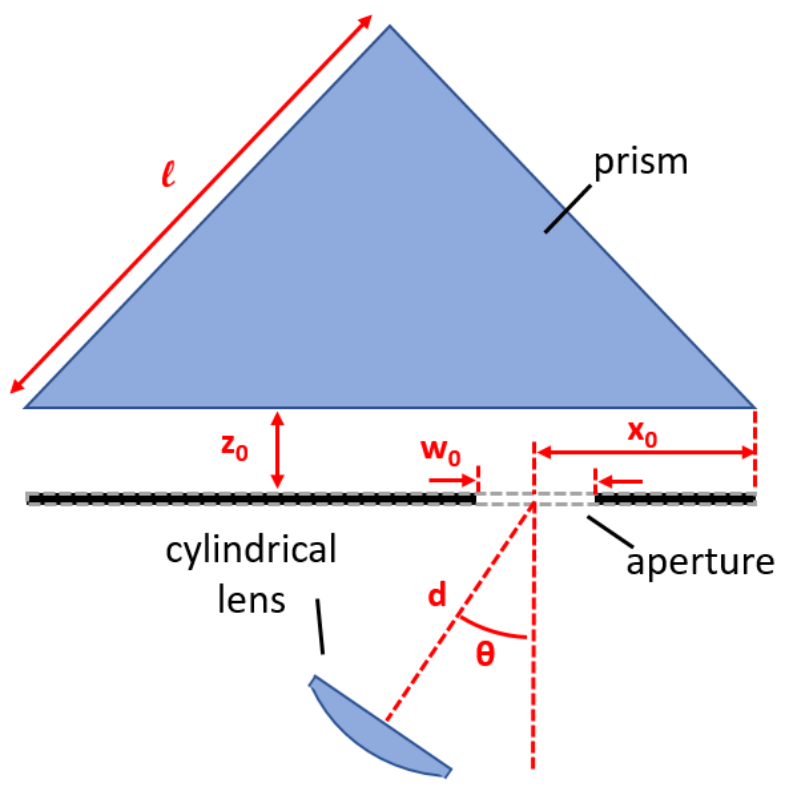

Figure 5: Depiction of the geometrical parameters for master VHOE fabrication. The lens position ' $\mathrm{d}$ ', and beam angle ' $\theta$ ', and aperture width ' $w_{0}$ ' are determined based on the desired properties of the array elements in the copy hologram and the prism face length ' 1 ', working distance ' $z_{0}$ ', aperture offset ' $\mathrm{x}_{0}$ ' are selected to appropriately position the coupling elements within the master VHOE.

\section{EXPERIMENTAL VALIDATION}

A prototype system was assembled using both commercial optical components and 3D printed parts designed in Solidworks to demonstrate the replication concept. The copy VHOE was mounted on two sets of motor controlled track stages for 2D lateral translation as shown in Fig. 6a. The stages were controlled using a Matlab script to provide the step-and-repeat procedure. The assembly for the master VHOE fabrication is shown in Fig. 6b. 


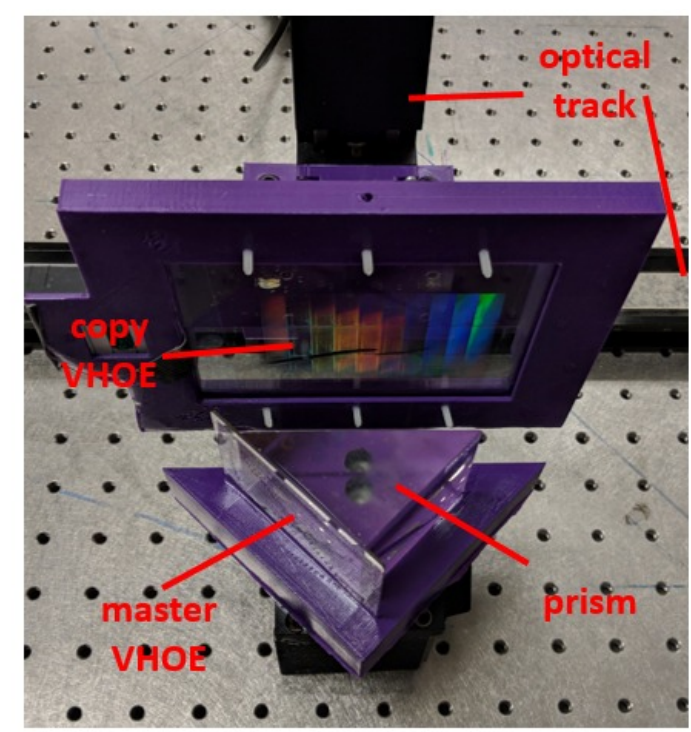

(a)

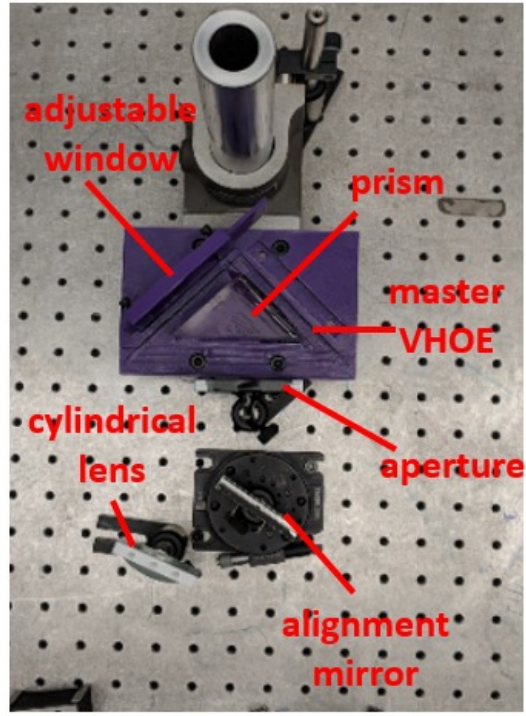

(b)

Figure 6: Optical setup for (a) fabricating the copy VHOE (b) fabricating the master VHOE.

Table 1: Parameters used for the experimental demonstration.

\begin{tabular}{l|l|l|l|l|l|l}
$\theta_{1}[$ degrees $]$ & $\theta_{2}[$ degrees $]$ & $\mathrm{D}[\mathrm{mm}]$ & $\mathrm{w}_{0}[\mathrm{~mm}]$ & $\mathrm{x}_{0}[\mathrm{~mm}]$ & $\mathrm{z}_{0}[\mathrm{~mm}]$ & $\boldsymbol{\ell}[\mathrm{mm}]$ \\
\hline 2.5 & 25 & 115 & 8 & 20 & 30 & 55
\end{tabular}

A holographic lens array was fabricated using the prototype system. The holographic material was Covestro Bayfol $\mathrm{HX}^{14}$ and the replication system parameters were determined using the design procedure described earlier and the resulting values are listed in Table 1. A total of 36 hologram array elements were recorded in the copy VHOE for total dimensions of $9.6 \mathrm{~cm} \mathrm{X} 6 \mathrm{~cm}$. The diffraction efficiency of each holographic lens was determined by measuring the spectral transmittance and assuming that all light that was not transmitted was diffracted in to the first order. Fresnel reflections and material absorption were accounted for by normalizing the transmittance curve to a portion of the photopolymer and substrate where no hologram was recorded. Each holographic lens had high diffraction efficiency and the median peak diffraction efficiency was $95.6 \%$ with a variability of $0.8 \%$. The spectral diffraction efficiency curves are presented in Fig. 7 and pictures of the holographic lens array are shown in Fig. 8. 


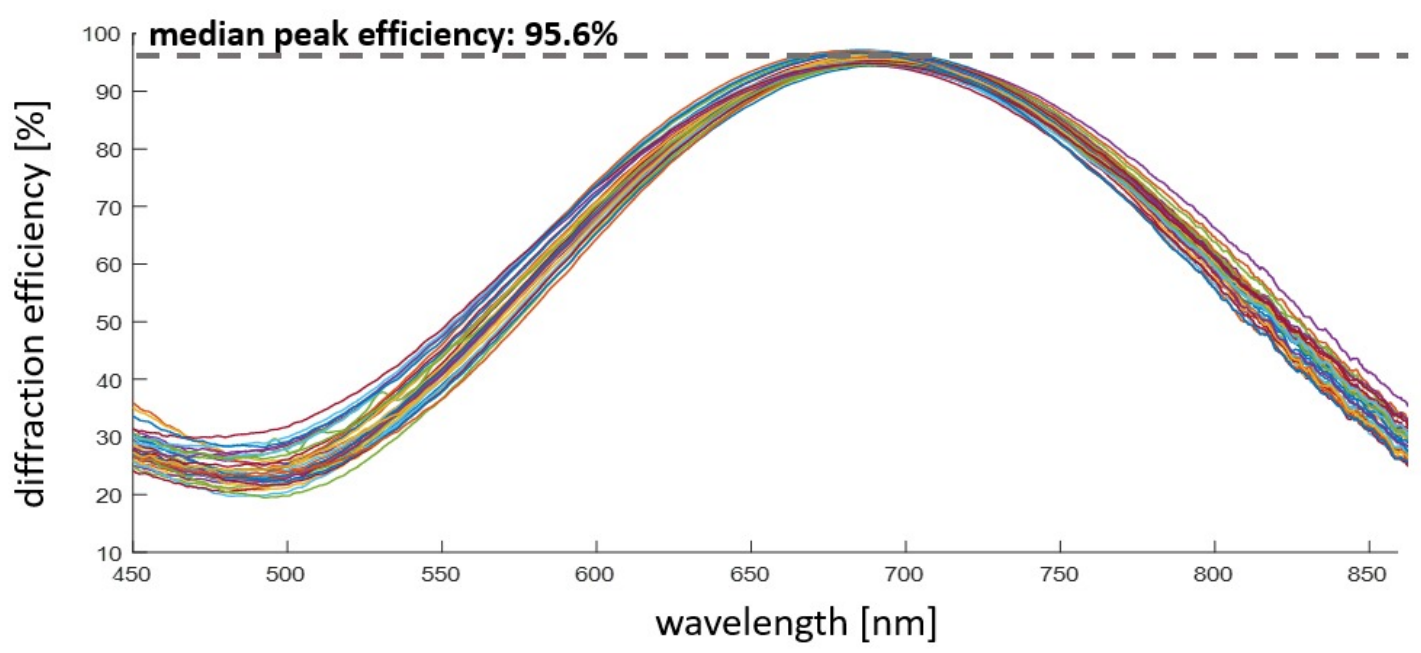

Figure 7: Measured spectral diffraction efficiency of each volume holographic lens in an array fabricated in Covestro Bayfol HX. The median peak diffraction efficiency was $95.6 \%$ with variability of $+/-0.8 \%$.

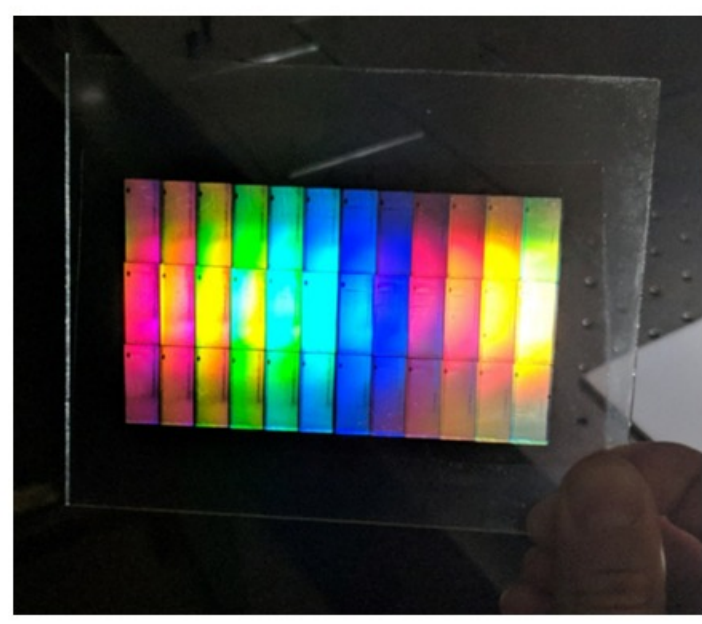

(a)

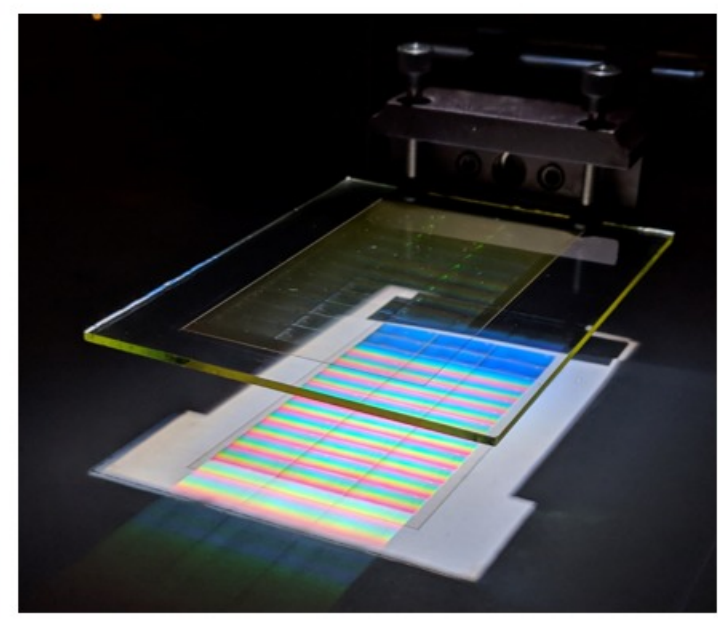

(b)

Figure 8: A 9.6cm X 6cm holographic lens array with 36 total holographic lenses that was fabricated in Covestro Bayfol HX.

\section{CONCLUSION}

In this paper we review a new replication system and method for fabricating hologram arrays. The method has advantages over the well-known contact copy based replication technique that make it well suited for manufacturing high-efficiency holographic lens arrays for spectrum-splitting photovoltaic systems. The system uses reference and object beam coupling elements in a master hologram to generate the holographic exposure for individual hologram array elements. The master hologram is in optical contact with a prism and the diffracted beams from the coupling elements are transmitted through the prism and overlap in the plane where the copy hologram is located. Light that is not diffracted by the master hologram is filtered by the prism through total internal reflection. A method for fabricating the master hologram and for determining the relevant system parameters is also reviewed. A prototype system was fabricated and used to make a high diffraction efficiency (95.6\%) holographic lens array with 
dimensions $(9.6 \mathrm{~cm} \mathrm{X} 6 \mathrm{~cm})$. The system can be implemented with inexpensive laboratory equipment, but also has potential for large-volume manufacturing of holographic elements for a variety of applications.

\section{ACKNOWLEDGEMENTS}

We would like to acknowledge support from our sponsors: NSF/DOE ERC cooperative agreement No. EEC1041895 and NSF grant ECCS-1405619. Ben Chrysler would like to acknowledge support from the National Science Foundation Graduate Research Fellowship Program Grant (DGE-1143953). Any opinions, findings, and conclusions or recommendations expressed in this material are those of the authors and do not necessarily reflect the views of the National Science Foundation.

The authors would like to thank Jianbo Zhao for allowing us to use his 3D printer for this study.

\section{REFERENCES}

1. Warmann, E. C., Flowers, C., Lloyd, J., Eisler, C. N., Escarra, M. D., Atwater, H. A., "Design of photovoltaics for modules with 50\% efficiency," Energy Sci. Engi. 5(2), 69-80 (2017).

2. Warmann, E.C., Atwater, H. A., "Energy production advantage of independent subcell connection for multijunction photovoltaics," Energy Sci. \& Engi. 4(4), 235-244 (2016).

3. Russo, J. M., Zhang, D., Gordon, M., Vorndran, S., Wu, Y., Kostuk, R. K., "Spectrum splitting metrics and effect of filter characteristics on photovoltaic system performance," Opt. Express 22(102), A528-A541 (2014).

4. Vorndran, S. D., Chrysler, B., Wheelwright, B., Angel, R., Holman, Z., and Kostuk, R., "Off-axis holographic lens spectrum-splitting photovoltaic system for direct and diffuse solar energy conversion," Appl. Opt. 55(27), 7522-7529 (2016).

5. Chrysler, B.D., Wu, Y., Yu Z., Kostuk, R.K., "Volume holographic lens spectrum-splitting photovoltaic system for high energy yield with direct and diffuse solar illumination," Proc. SPIE 10368, 103680G (2017).

6. "National Solar Radiation Data Base: 1991-2005 Update: Typical Meteorological Year 3," NREL. Retrieved 18 February 2016. 〈http://rredc.nrel.gov/solar/old_data/nsrdb/1991-2005/tmy3/〉.

7. Bruder, F., Fäcke, T., Grote, F., Hagen, R., Hönel, D., Koch, E., Rewitz, C., Walze, G., Wewer, B., "Mass production of volume holographic optical elements (vHOEs) using Bayfol ${ }^{\circledR} \mathrm{HX}$ photopolymer film in a roll-to-roll copy process," Proc. SPIE 10127, 101270A (2017).

8. Tipton, D. F., "New hologram replicator for volume holograms and holographic optical elements," Proc. SPIE 3294, 136-145 (1998).

9. Aspnes, E. D., Castillo, J. E., Courreges, R. D., Hauser, P. S., Rosenberg, G., Russo, J. M., "Volume hologram replicator for transmission type gratings," U.S. Patent 8,614,842, issued December 24, 2013.

10. Stojanoff, C. G., "Review of the technology for the manufacturing of large-format DCG holograms for technical applications," Proc. SPIE 3011, 267-279 (1997).

11. Okui, M., Wakunami, K., Oi., R., Ichihashi, Y., Jackin, B. J., Yamamoto, K., "Copying of holograms by spot scanning approach," Appl. Opt. 57(15), 4117-4122 (2018).

12. Saxby, G., [Practical holography], CRC Press, (2003). 
13. Chrysler, B.D., Kostuk, R.K., "Volume hologram replication system for spectrum-splitting photovoltaic applications," submitted July 10.

14. Marin-Saez, J., Atencia, J., Chemisana, D., Collados, M., "Characterization of volume holographic optical elements recorded in Bayfol HX photopolymer for solar photovoltaic applications," Opt. Express 24(6), A720-A730 (2016).

Proc. of SPIE Vol. 10759 107590W-10 